\title{
Review
}

\section{Laser acceleration and its future}

\author{
By Toshiki TAJIMA*1,*2,†
}

(Communicated by Toshimitsu YamazAKI, M.J.A.)

\begin{abstract}
Laser acceleration is based on the concept to marshal collective fields that may be induced by laser. In order to exceed the material breakdown field by a large factor, we employ the broken-down matter of plasma. While the generated wakefields resemble with the fields in conventional accelerators in their structure (at least qualitatively), it is their extreme accelerating fields that distinguish the laser wakefield from others, amounting to tiny emittance and compact accelerator. The current research largely falls on how to master the control of acceleration process in spatial and temporal scales several orders of magnitude smaller than the conventional method. The efforts over the last several years have come to a fruition of generating good beam properties with $\mathrm{GeV}$ energies on a table top, leading to many applications, such as ultrafast radiolysis, intraoperative radiation therapy, injection to X-ray free electron laser, and a candidate for future high energy accelerators.
\end{abstract}

Keywords: laser acceleration, collective fields, relativistic coherence, wakefields, collective deceleration

\section{Introduction}

The Livingstone chart ${ }^{1), 2}$ shows the energy of various generations of accelerators exponentiates over the years ever since its incipience. However, in recent years it appears that this steady growth seems to have ceased and less robust growth is seen. This tendency is attributed to the lack of major new ideas since 1960's according to Ref. 3. In lack of major new ideas the only way to increase the energy is either by finessing the conventional technologies, or by increasing the size (and budget) exponentially. This faces a severe prospect that the society is now saddled with to pay a substantial sum for accelerators in ultra high energies. The author personally experienced a devastation at the demise of Superconducting Super Collider (SSC), ${ }^{4)}$ as he had been intimately involved in the SSC accelerator physics

*1 Faculty of Physics, Ludwig-Maximilian University, Garching, Germany (also at Max Planck Institute for Quantum Optics, Garching, Germany; Kansai Photon Science Institute, JAEA, Kyoto; and KEK, Ibaraki, Japan).

*2 Blaise Pascal Chair, Ecole Normale Superieure of France.

$\dagger$ Correspondence should be addressed: T. Tajima, Faculty of Physics, Ludwig-Maximilian University, am Coulombwall 1, 85748 Garching, Germany (e-mail: toshiki.tajima@physik.uni-muenchen.de). research as a faculty member of the University of Texas at Austin at that time. Even the richest country in the world was unable to sustain the SSC accelerator any more.

Very crudely, the attainable maximum energy is proportional to the size of the accelerator and so is the cost. SSC tried to achieve $20 \mathrm{TeV}$ protons in energy with the circumference of $10^{2} \mathrm{~km}$. This is due to the fact that the basic building blocks of the accelerator, the rf acceleration cavity or the bending magnet, are limited by the highest field that can be achievable. In the case of the rf accelerating cavity, the accelerating gradient is limited by the metallic (material) breakdown field. It is currently much less than $100 \mathrm{MeV} / \mathrm{m}$. Beyond this field, the material sparks and electrically break down. One may estimate such a breakdown field by saying that the electron in the metal may dislocate to other site by the energy barrier on the order of the atomic energy, say $1 \mathrm{eV}$. Such a dislocation is over an Angstrom, $10^{-8}$ $\mathrm{cm}$, which yields the field at $100 \mathrm{MeV} / \mathrm{cm}$, some two orders of magnitude higher than the actual breakdown field of the material. This is due to the imperfection and impurities of the material and its associated electron avalanche phenomenon in real materials. Thus, as long as we employ such mate- 
rials, we are limited by the breakdown field and the necessary size in accordance. Jared Diamond ${ }^{5}$ has analyzed the pattern of demise of many (isolated) societies (so that its demise may be isolated, rather than the destruction by external forces such as war) and concluded that the reason for the collapse of one particular civilization, invariably, is due to the destruction of its environment. For example, he cites the famous example of the Easter Island, where its civilizational goal was to erect a huge stone stature of each tribe's chieftain. As time went on, chieftains wanted to erect an ever bigger stature with more woods to cut to prepare for it, forcing less and less woods to remain. Even the last tree was cut in order to promote this civilizational goal, since then there was no more wood, leading to civil wars and collapse of this civilization. The scientific and technological progress of the 20th Century has been remarkable, while its commanding resources to sustain its ever more ambitious leap have become also non-negligible to the global scale. We certainly wish to learn from Diamond's thesis and not to repeat the kind of collapse that the Easter Island had experienced.

Veksler introduced the idea of collective acceleration using plasma in 1956. ${ }^{6}$ By introducing plasma as the accelerating medium, he liberated the accelerator physicist from the electrical breakdown of media that have inflicted the saturation of the upper limit of the accelerating gradient. His idea was to inject an electron beam into plasma to exert a large amplitude plasma wave to accelerate heavier ions, so that he hoped that the ion energy gains as much as the mass ratio $M / m$ over the driving electron energy, where $M$ and $m$ are the masses of ions and electrons. The fields excited in the plasma are of collective oscillations of plasma that can be far surpassing over those of the individual particle nature, thus the name of collective acceleration. Typically, instead of the accelerating field independent of the number of particles $N$ when the accelerating field originates from individual nature, the collective fields are proportional to $N$. However, the research that ensued to demonstrate his idea showed that his idea introduced new problems as well. ${ }^{7}$ In order to trap nonrelativistic ions, the electrons need to become nonrelativistic ('reflexing'), in which case the electron-triggered plasma wave becomes unstable leading to the trapping bucket for ions quickly broken. Mako and Tajima ${ }^{8)}$ analyzed the nature of this process on their own model.9) Their analysis shows that the ion energy gain is mere several times the electron energy in contrast to the expectation of Veksler by $M / m$, due to the plasma wave breakup.

In 1979 Tajima and Dawson ${ }^{10)}$ introduced the concept of Laser Wakefield Acceleration (LWFA). A short intense laser pulse with appropriate pulse length is injected into a plasma with the appropriate density can excite a plasma wave that is its own resonant eigenmode. We showed that this resonant wave can attain large amplitude (we call relativistic amplitude) such that the electron motion in the wakefield acquires relativistic energies. This wave constitutes a fairly robust structure moving with a relativistic phase velocity, which renders the electron overtaking more difficult (thereby resisting the wave breaking) and thus the electron accumulation more manifest, exhibiting a nonlinear entity of density cusp singularity. We tried to circumvent the fragility of the wave associated with nonrelativistic phase velocity by making the LWFA at relativistic phase velocity. We avoided the narrow trapping width of ions due to its heavy mass as analyzed, ${ }^{8)}$ by the adoption of accelerating electrons. This last point becomes important when we discuss laser ion acceleration, but we will not enter this topic in any further detail, leaving this discussion for a recent review elsewhere. ${ }^{11)}$ These safeguards should overcome the difficulties that were encountered in the Veksler concept. In the end the Laser Wakefield Acceleration accomplishes the rectification of fast-oscillating large transverse laser fields that cannot contribute to acceleration (dictated by the Woodward-Lawson Theorem $)^{12)}$ into fast-oscillating large longitudinal fields that are fit for particle acceleration.

In the following we expound on this LWFA concept and its realization in more detailed accelerator context and experimental settings. We also characterize the properties of LWFA and overview some of the emerging applications.

\section{Laser wakefield acceleration}

A charged particle moving in a plasma is capable of causing a wake behind ${ }^{13)-15}$ ) steeped in the modern statistical physics framework. ${ }^{16)}$ As mentioned above, this is the elementary process that Veksler6) tried to exploit, except in its collective version beyond the individual particle version. ${ }^{14), 15}$ The wake may be regarded as a Cherenkov radiation of plasma waves behind the speeding charged par- 

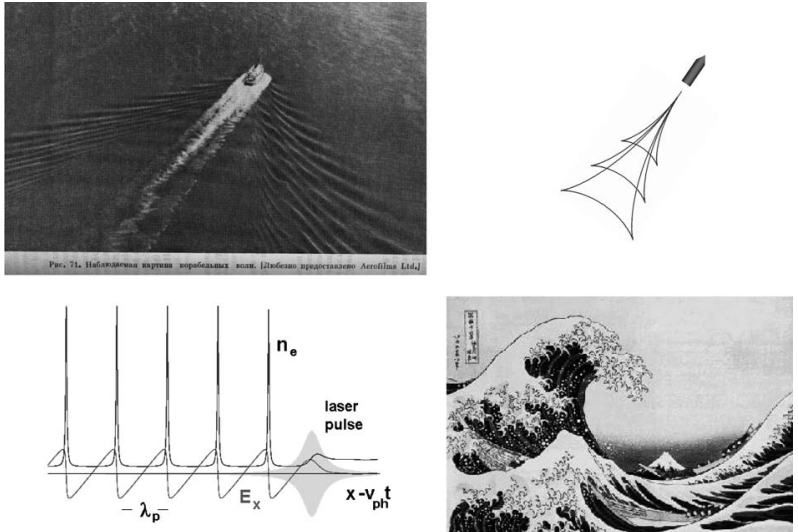

Fig. 1. Wakefield and the wave dynamics in the large amplitude, a collective phenomenon. The upper frames show both a boat leaving a $2 \mathrm{D}$ wakefield behind on the lake water surface in a photograph (left) and its schematics of Kelvin wake (right). The lower left frame shows a 1D simulation of a laser pulse speeding to the right leaving behind a largeamplitude plasma wakefield $\left(E_{x}\right)$ and density modulations exhibiting a cusp singular structure. No wave breaking is seen in this relativistic laser plasma dynamics. On the other hand, the lower right shows a large-amplitude water wave, whose phase velocity is non-relativistic and showing wave breaks and white waters, famously captured by the Ukiyoe by Hokusai. (by courtesy of S. Bulanov).

ticle. See Fig. 1. In Veksler's case when a large collection of charged particles in the electron beam is injected into plasma and the collective excitation of large plasma waves are excited, their back reactions are not negligible, which tends to retard some of the electrons back. (A case where the electron bunch is a long beam and gives rise to a beam-plasma instability that leads to enhanced fluctuations similar to the phase transition critical slowing-down was considered. ${ }^{13)}$ ) And thus the electrons cannot be treated as test particles any longer. In the example that was analyzed, ${ }^{8)}$ the head of the electron beam was ejected forward (i.e. accelerated) and ran away, while the rest of the electrons were pulled back (called the 'reflexing' motion ${ }^{8)}$ ) toward where ions were before they were yet to be highly accelerated. This breakup of electrons into the categories of being ejected and reflexed is due to the inertia of ions and their slow velocity compared with the electron beam. The excited plasma wave was unable to trap ions, because the trapping width ${ }^{17)}$ of this plasma wave was not sufficiently large to trap the slow ions from the relativistic electron speed.
Tajima and Dawson proposed the employment of a short and intense laser pulse to excite a wakefield in such a way that the laser pulse length $\sigma_{0}$ is resonant to the wavelength of the eigenmode of the plasma, i.e. half of the plasma wavelength $\lambda_{p}$ :

$$
\sigma_{0}=\lambda_{p} / 2 .
$$

The recommended intensity of the laser pulse is such that the pondermotive potential (the photon pressure force potential) of the laser in the plasma amount to

$$
\Phi=m c^{2} \sqrt{\left(1+a_{0}^{2}\right)},
$$

so that the excited plasma wave motion acquires the electron momentum of $m c a_{0}$. Here the normalized vector potential of the laser is $a_{0}=e E_{0} / m \omega_{0} c$ and $E_{0}, \omega_{0}$ are the electric field and frequency of the laser. The ponderomotive force arises from the nonlinear Lorentz force $v \times B / c$, which causes the polarization of electrons in the plasma in the longitudinal direction, even though the electric field of the laser was in the transverse direction. This polarization

$$
E_{p}=m \omega_{p} c a_{0} / e,
$$

yields the electrostatic field in the longitudinal direction in turn in the same magnitude. This is the origin of the excited wakefield. See Fig. 1. When the laser is at the verge of relativistic strength, i.e. $a_{0}=1$, the wakefiled amplitude assumes the value of

$$
E_{w b}=m \omega_{p} c / e,
$$

which is the wave breaking field in the non-relativistic case. ${ }^{18)}$ The wave tends to break if the wave amplitude is high so that the high amplitude portion of the wave typically propagates faster than the lower portions and takes over those. As seen in Fig. 1, the famous Hokusai's Ukiyoe illustrates the way the nonrelativistic large amplitude ocean waves break. This $a_{0}=1$ condition is realized, for example, when the laser intensity is in the neighborhood of $10^{18} \mathrm{~W} / \mathrm{cm}^{2}$ for $1 \mu \mathrm{m}$ laser. The wave breaking field [4] is as great as $1 \mathrm{GeV} / \mathrm{cm}$ at the plasma density of $10^{18} / \mathrm{cc}$. This field or equivalently [3] is several orders of magnitude greater than the breakdown field when the cavity begins to spark and thus can contribute to compactifying the accelerator by several orders of magnitude over the conventional ones. It is important to further notice that the wave breaking field (in nonrelativistic regime) is a bit of misnomer, as the relativistic wakefield resists the wave break, as 

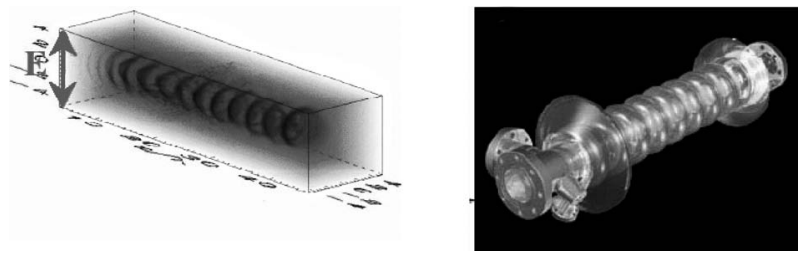

Fig. 2. Thousand-fold compactification of accelerator. The comparison of the structure of the induced LWFA in a plasma (a) and the superconducting waveguide (b). Though the structures are not so distinct, the size of the accelerators are at least thousand times smaller in LWFA than the conventional one. (a) The entire box is about $0.1 \mathrm{~mm}$ with the accelerating field of $10^{5} \mathrm{MV} / \mathrm{m}$, while (b) the superconducting linac tube is about $40 \mathrm{~cm}$, providing the accelerating field of $32 \mathrm{MeV} / \mathrm{m}$ (Fermilab). (by courtesy of T. Esirkepov).

electrons cannot easily overtake those in less momenta, as the wave is propagating at a relativistic speed and electrons have harder time to take over this speed because they cannot exceed the speed of $c$. Instead they pile up at the particular wave phase to form a cusp singularity. ${ }^{19), 20)}$ Thus relativity facilitates matter to form robust structures and also enhances the maximum ('wave breaking') field above which they break, leading to much greater accelerating gradient in the relativistic regime ${ }^{21)}$ (see Fig. 1). Thus relativity serves as the guiding principle to regulate and increase the wakefield. This relativistic coherence has been exploited for what we call 'relativistic engineering', in which we take advantage of the coherent and stable properties arise because of the matter's tendency to cohere toward the speed of light. ${ }^{22), 23)}$ The laser wakefield is a collective field that is generated in plasma involving all the electrons of the plasma in a coherent fashion, forming robust resonant oscillations stabilized and enhanced by its relativistic dynamics. The amplitude of the wave [3] is by many orders of magnitude greater than the wake generated due to the individual electron beam particle calculated. ${ }^{13), 14)}$ If the wave has the amplitude of [4], electrons can be picked up from the rest to attain the momentum of $m c$, or the trapping velocity width is $c$. This is why the wave near the wave-breaking limit is capable of scooping up electrons that are at rest. It is retrospectively of interest why ions were not easily trapped by the Veksler's plasma wave excited by the electron beam, because the trapping width for ions is $\sqrt{(m / M)}$ times smaller than the electron trapping mentioned here. When energetic electrons exceed the speed of ions plus the ion trapping width of the wakefield $v_{i}+c \sqrt{(m / M)}$, which is quite small, they are ejected from the potential bucket and run away from ions. Thus the laser electron acceleration by wakefield overcomes the Veksler's difficulty.

The laser wakefield accelerator can thus be thousand-fold or more compact (or with thousandfold, or more intense accelerating gradient) than the conventional best rf accelerator. This is depicted in Fig. 2. Beside this stark size difference, the two share a rather common field structure as also seen in Fig. 2. Unique in LWFA is the dephasing between the high energy electrons and the laser due to the plasma permeability. (In the conventional rf accelerator, the waveguide modes that have the phase velocity greater than $c$ is slowed down to match the speed of light $c$ by instituting the slow-wave structure.) The phase velocity of the wakefield

$$
v_{p}=c\left(1-\gamma_{p}^{-2}\right),
$$

where the relativistic Lorentz factor becomes $\gamma_{p}=\omega / \omega_{p}{ }^{10)}$ For more discussion see Ref. 23. This leads to the length over which the wakefield slips from the electron over half the wavelength so that no longer able to continue acceleration:

$$
L_{d}=\lambda_{p} \gamma_{p}^{2} .
$$

Over this length electrons gain energy by

$$
\Delta \varepsilon=2 \gamma_{p}^{2} m c^{2} a_{0}^{2} .
$$

Here the factor $\left(a_{0}^{2}\right)$ is multiplied when the laser intensity is high $\left(a_{0} \gg 1\right)$. In the case of $a_{0} \gg 1$, the laser depletes its pump energy much faster than it dephases. ${ }^{24)}$ The basic way to overcome these problems of dephasing and pump depletion before reaching high energies is to break up the acceleration stage into a multiple of stages, in each of which the relative longitudinal position between the particle and laser is readjusted. ${ }^{12)}$ The acceleration in a plasma fiber may also contribute to the adjustment of the phase velocity. ${ }^{12)}$ See Fig. 3. Some more issues regarding the multistaging were discussed. ${ }^{25)}$

\section{Experimental realization}

The required shortness of the laser pulse as well as the requested intensity to reach the neighborhood of the singular value of the LWFA with robustness (as seen in Fig. 1) did not exist at the time of Tajima and Dawson's paper in 1979. Most of the lasers with large energy were for laser fusion experiments, which 

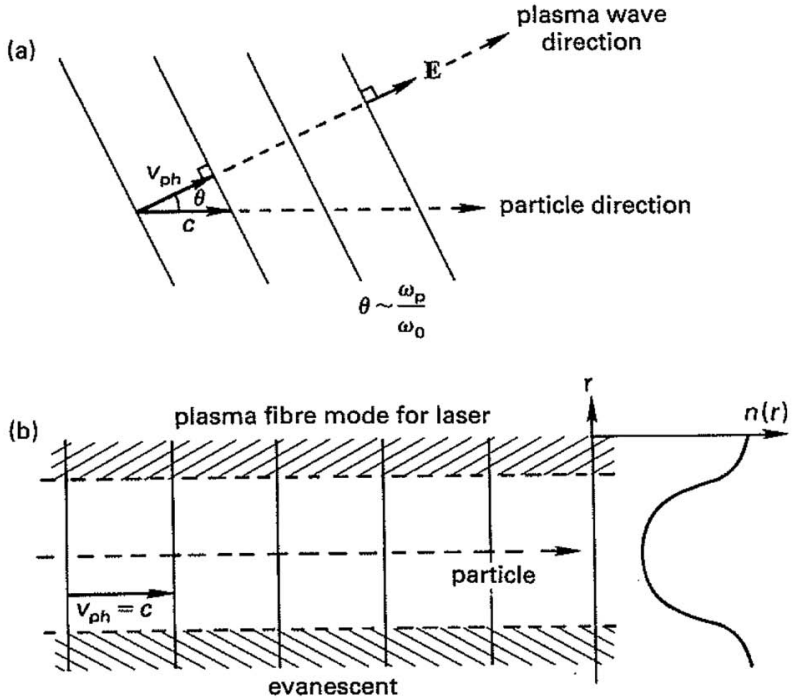

(c)

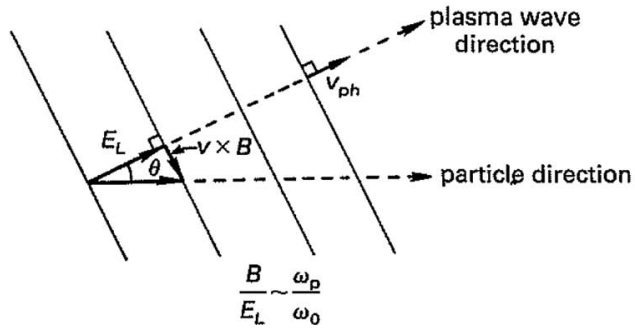

Fig. 3. Plasma fiber acceleration: The laser tends to diverge over the Rayleigh length $Z_{R}$. However, the laser may be confined by a plasma fiber, in which the plasma density is low inside than the surrounding area (b); inside the fiber the angle between the particle propagation and the wave vector may be present (a); the presence of magnetic field ( $B_{\theta}$, induced by plasma and/or externally imposed) may compensate for the angled acceleration and also for the dephasing of the phase velocity $\left(v_{p}<c\right.$, see Eq. [5]) of the accelerating field (c) with particle velocity. (after Ref. 12).

had typically the ns duration. These never approached the fs time regime nor the $10^{18} \mathrm{~W} / \mathrm{cm}^{2}$ intensity where the electron dynamics in the laser field becomes relativistic. However, the invention of Chirped Pulse Amplification (CPA) started in 198526) and subsequent efforts by Mourou et al. has changed all that. This method quickly introduced optics in the relativistic regime. ${ }^{20)}$ By mid-1990's the laser intensity has reached the relativistic regime with CPA in more than one labs around the world with fs time scales. Thus experimental realization of what Tajima and Dawson proposed advanced rapidly in 1990's. On the way many ultrafast techniques and phenomena had to be learned, while copious new phenomena in the relativistic regime of laser and laser-matter interaction needed to be discovered and disentangled. After all, the wavelengths of lasers are several orders of magnitude shorter than the counterparts of the conventional rf cavity waves. This meant that we had to be able to conduct experiments with several orders of magnitude more accurate, precise, and finessed control. Earlier experiments relied on stopgap remedies such as the beat wave, or the selfmodulated wakefield excitation, which led to broad energy spectra (see review ${ }^{24)}$ ). Once experimentalists enter the regime with a short enough laser pulse [1] and relativistic intensity, they found that the LWFA can much cleanly pick up electrons than experiments till then. This was largely predicted in the original paper, but in addition it was facilitated by the wave breaking in $2 \mathrm{D}$ (or $3 \mathrm{D})^{27), 28)}$ that arises easily as in the nonrelativistic regime of $1 \mathrm{D}$, while the $1 \mathrm{D}$ relativistic wake is robust against wave break, as mentioned above. Then there emerged electron spectra with nearly monoenergy. ${ }^{29)-31)}$ Later even a $\mathrm{GeV}$ electron beam with monoenergy spectrum was produced over a few centimeters. ${ }^{32)}$ See Fig. 4. In this experiment the experimentalists ${ }^{32}$ employed the plasma density at a few times of $10^{18} / \mathrm{cc}$ with a $40 \mathrm{TW}$ laser (focused to yield the laser intensity in the neighborhood of $10^{18} \mathrm{~W} / \mathrm{cm}^{2}$ ). This experiment is remarkable in many ways. It is the first LWFA experiment to reach GeV over mere few centimeters. Just to mention another of these, this happened to operate almost exactly in the same ballpark of the parameter regime suggested. ${ }^{10)}$ Furthermore, the consequent results of the experiment, such as the strength of the accelerating gradient, closely parallel with the prediction. ${ }^{10)}$

Since then, many subsequent experiments have pushed the frontier of the science and arts of LWFA tremendously. In my current home of Max Planck Institute for Quantum Optics (MPQ), the scientists have perfected the LWFA to become reproducibly and repeatably monoenergy (Osterhoff et al.). ${ }^{33)}$ See Fig. 5. Other colleagues have utilized a few cycle (less than 10 fs extreme short pulse) laser to produce LWFA accelerated electron bunches that have no (or little) bulk portion at all, showing only the beam component. ${ }^{34)}$ See Fig. 6. Our Korean colleagues have gone $\mathrm{GeV}$ in a stable generation mode in a gas jet, ${ }^{35)}$ while our Japanese colleagues have explored to increase the stability and controllability of the laserdriven beam generation. ${ }^{36), 37)}$ 


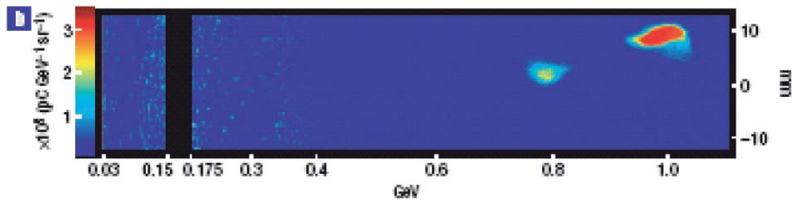

Fig. 4. GeV monoenergetic electron acceleration demonstrated. ${ }^{32)}$ A narrowly clumped electron bunch is concentrated near the GeV energy in this experiment. The acceleration took place over a $3 \mathrm{~cm}$ plasma fiber (channel). (by courtesy of W. Leemans).

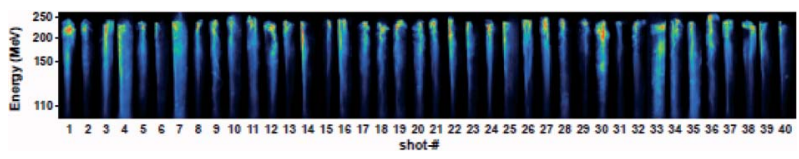

Fig. 5. Reproducible LWFA was demonstrated. ${ }^{33)}$ From laser shot to shot, each time the LWFA accelerates electrons to a narrow energy bunch with the same peak energy with a similar energy spread. (by courtesy of S. Karsch).

\section{Selected applications}

The advance in the LWFA experimental verifications and in mastering the experimental finesse as discussed in the previous section has allowed to portend and realize a broad range of its applications utilizing the LWFA unprecedented characteristics. These applications range from ultrafast (fs) radiolysis, compact X-ray and $\gamma$-ray sources, cancer therapy, Xray free electron laser injector, compact radioactivation machines such as PET sources, calibration of high energy accelerators and cosmic ray detectors, compact bunch decelerators, compact bunch choppers and plasma lens of charged particle beams, relativistic engineering in general including photon beam compression, etc. in addition to high energy accelerators themselves including a future collider candidate. For example, in laser driven ultrafast radiolysis, Malka's group $^{38)}$ and Crowell et al. ${ }^{39)}$ have carried out pioneering works in this field. Here, however, let us dwell on but two examples at some length.

The first example is an application of LWFA to intense (and possibly coherent) X-ray generation. My colleagues at MPQ are spearheading this effort. ${ }^{40)}$ The electron bunch created by LWFA is much less than $\lambda_{p}$ in the longitudinal direction in size and less than the laser spot size $\sigma_{x}$ (where $x$ is the transverse direction), which are both many orders of magnitude smaller than those in conventional accelerators. Since the number of particles in a bunch is either

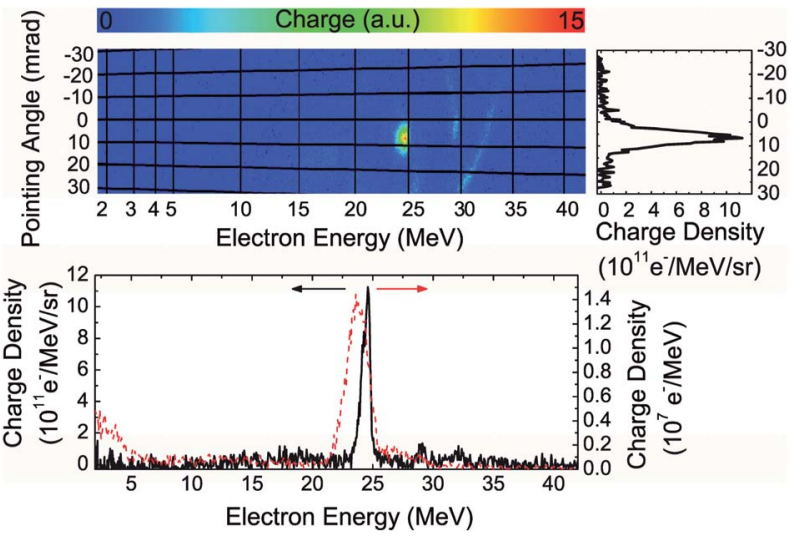

Fig. 6. Monoenergy electrons from a few cycle laser was demonstrated. ${ }^{34)}$ The LWFA generated electron bunch is sharply peaked both the transverse dimensions as well as the longitudinal dimension. Its energy is peaked with little tail electrons visible. (by courtesy of L. Veisz).

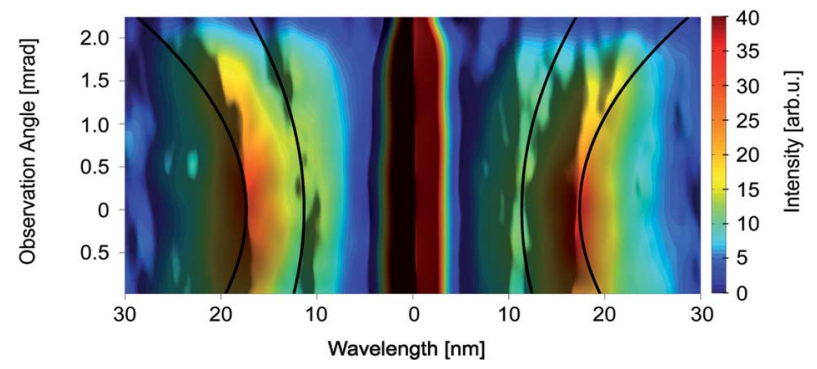

Fig. 7. X-ray undulator radiation driven by LWFA generated electron bunches, as shown by Ref. 40. X-rays have been generated over mere tens of $\mathrm{cm}$ of the magnetic undulator. (by courtesy of F. Gruener).

comparable or slightly less than that of the conventional accelerators, the current, or the density of the beam of LWFA is much higher than the conventional ones. This property makes the LWFA beam as a source for radiation, in general, very attractive, as it can generate brilliant electromagnetic radiation, be it the betatron radiation from electrons oscillating in the laser wakefields themselves, or synchrotron radiation in a bending magnets, matter, or in undulators (see recent progress ${ }^{41}$ ). The free electron laser radiates if the electron bunch can form coherent radiation from undulators. In Ref. 40 they inject the LWFA bright (and compact) bunch into undulators, getting so far incoherent $\mathrm{X}$-rays from relatively short (tens of centimeter undulators). See Fig. 7. With less energy spread (and other improvements) it is expected to lase over a length that is much smaller 


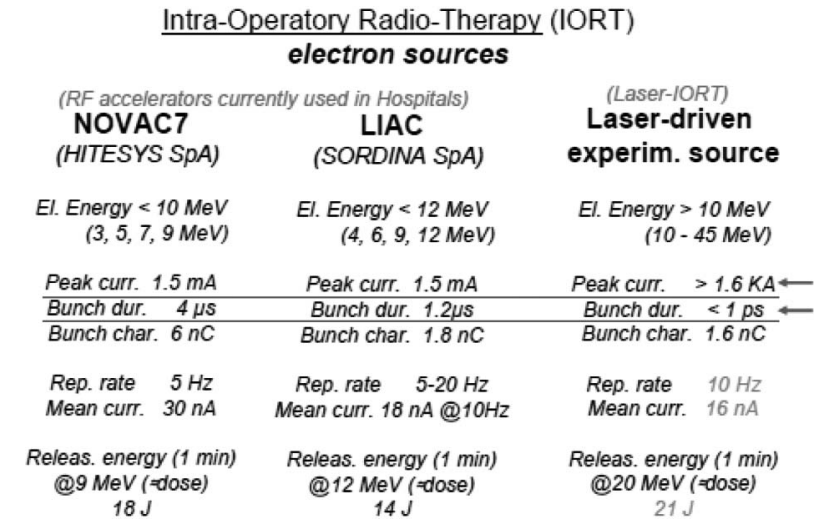

Fig. 8. Compact Intra-Operative Radio Therapy (IORT) and gamma-ray radiolysis driven by LWFA have been studied. ${ }^{46)}$ The experimental comparison shows that the average parameters of the beam are similar between the conventional source and the LWFA driven one, while the compactness and pulsed nature are quite disparate. (by courtesy of A. Giulietti).

than the conventional XFEL undulator length. The fact that the LWFA bunch is much more compact leads to much more brilliant X-rays and perhaps more coherent. This is because the collective and therefore coherent radiation occurs when the wavelength can be longer than the bunch size. Once this occurs, the radiation power is proportional to $N^{2}$, as compared to incoherent radiation proportional to $N$, where $N$ is the number of electrons that emit the radiation. Coherent X-ray sources based on this or on relativistic engineering ${ }^{22)}$ (or other methods) can, for example, enable us to introduce phase sensitive physics research and X-ray measurement techniques, such as the Phase Contrast Imaging. ${ }^{42)}$ The laser wakfield is also known for excellent and strong source for coherent $\mathrm{THz}$ radiation, ${ }^{43), 44)}$ which in turn may be employed for biological tissue analysis, material analysis, detection of clandestine materials, art conservation $^{45)}$ etc. In short LWFA beams have far stronger penchant for brilliant radiation than the conventional accelerator beams.

The second example is applications to medical radiology. The LWFA can deliver sufficiently high energy and high dose of electron beams in a very compact setting. Utilizing this feature Giulietti et $a l .{ }^{46)}$ have demonstrated the scenario of delivering electron beam based radiation for the Intra-Operative Radiation Therapy (IORT) of tumors derived from their experimental data. The peak current of these laser driven accelerators is one million times higher than with conventional RF-driven accelerators at the same dose rate. The crucial advantage is its compactness and versatility during the operation. ${ }^{47)}$ Giulietti et al. have further envisaged the application of LWFA to yield enough dose to get, via bremmstrahlung in a suitable dumper, relevant flux of gamma-rays in the spectral range of the Giant Dipole Resonance. These latter were efficiently used to produce via photo-activation radioactive isotopes. See Fig. 8. A European patent has been submitted on the invention of IORT based on LWFA.

These are only two examples among many recent application advances. Nonetheless, they show that LWFA is providing the transformative technology for desperate fields of importance. Speaking of the advantage of LWFA characteristics, let us mention here that the shortness of the laser wakefield generated beams allow unprecedented advantage in stopping such beams after the usage. As the accelerators accelerate electrons higher energies, these accelerated electrons after use become a headache to stop and dump. This is because the stopping range of high energy electrons rapidly increases as a function of energy. Another problem is that these high energy beams when dumped in the solid matter, they become radioactive and then become hard to dispense with. Finally, as the energy of the beams gets higher, it suffers less and less collisions (of course, this is why it takes a long distance to stop, as above). It also means that we are really wasting a majority of the beam energy each time we accelerate it to higher energies. The method of collective deceleration ${ }^{48), 49)}$ alleviates all these three problems simultaneously. However, the collective deceleration applies only to ultrashort bunches, whose bunch length is less than the plasma wavelength. This implies that LWFA generated beams are ideally matched for the collective deceleration and thus LWFA not only affords a compact accelerator, but also provides conditions for a compact, clean and efficient collective decelerator (in place of a radioactive large beam dump).

\section{The future of laser acceleration}

The laser wakefield accelerator research is progressing very rapidly these days. First, it is increasing the electron energy and now reaches beyond $\mathrm{GeV}$. It may soon see energies of $10 \mathrm{GeV}$ without new experimental setups. Beyond that, one may need some more new steps, either by laser or by the 


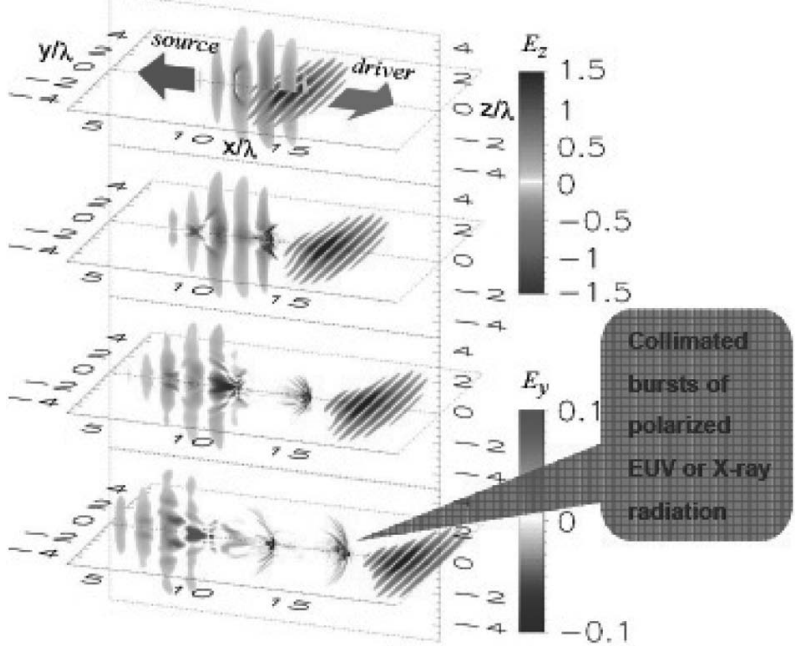

Fig. 9. LWFA boosts laser to extreme fields, as suggested by Ref. ${ }^{22}$ ) The LWFA driven wake has the divergingly large density peak (the cusp singularity), which can act as the Einstein's relativistic flying mirrors. The coherent X-rays backscattered from the counterstreaming laser on the mirrors may be focused by the concave geometry of the wakefield. Attosecond and even zeptosecond X-ray pulses with the Schwinger intensity have been suggested utilizing the relativistic dynamics of LWFA. A first tested relativistic engineering example. ${ }^{57)}$ (after Ref. 22).

multi-stage setups, and other improvements. These are energy advance. The other front is to improve the beam quality such as emittance, energy spread, repeatability, reliability etc. Since the laser wavelength is more than three orders of magnitude shorter than that of rf, the accuracy and finesse necessary are that much more difficult and demanding. It will take a large amount of learning and innovation to accomplish these, though there is no fundamental problem foreseen. The spread and rooting of ever more ingenious applications of LWFA are very important. Perhaps, we have not yet seen most exciting applications of LWFA as of today. Nonetheless, it is already clear that applications that entered so far are not just a compact version of the conventional accelerators. Some are exploiting unique properties of LWFA to unique problems.

As to the high energy accelerator, the liner collider based on the conventional rf technology has been envisaged. While this research is vigorously on going, its cost is proportional to the length of the accelerator, and because of the constraint that the accelerating gradient is upper-limited in the conventional technology, proportional in turn to the energy aimed at according to Raubenheimer. ${ }^{50)}$ When we aim at $\mathrm{TeV}$ energies, the length for such a linear collider becomes over 10 's $\mathrm{km}$, translating into a cost much beyond several billions of dollars, an order of magnitude (or more) costlier than any other linear colliders. The LWFA gradient is two to four orders of magnitude greater than the highest of the conventional rf technology, so that the potential cost saving is really significant. Currently, the biggest challenges are: (i) if the laser that drives the collider can come with enough fluence and efficiency (Mourou has organized a conference on such a question and provided a tentative affirmative answer through the fiber laser technology ${ }^{51)}$ ) and other activities encompassing over the laser and accelerator communities;52) (ii) if the laser acceleration method can master all the necessary intricacies that amount to the required elements of a collider. The first collider study based on LWFA was carried out by Xie et al. in 1997. ${ }^{53)}$ And finally, there seems to be no alternative path other than LWFA to reach for ultimately high energies such as $\mathrm{PeV},{ }^{54)}$ regardless of efficiency or luminosity.

In terms of its ultrafast (or ultrabright) aspect of LWFA, the applications such as to the XFEL mentioned in Sec. III are typical. There are many applications that are based on this property. And more are easily imaginable to come.

In terms of LWFA's compactness, other than the above high energy accelerators, we mentioned above the Intra-Operative Radiation Therapy (IORT) as an example. ${ }^{46)}$ Many such applications are envisioned and we deem this as possible.

Another characteristics of LWFA is its obvious synchronization with the laser and its precise control with the generated electron bunch with the laser. Because of this property, we envision that highly sophisticated cross-fertilization between the laserdriven electron bunched and the laser pulses to create a new class of X-rays and other quantum beams. Laser Compton scattered gamma rays are but such an example. ${ }^{55}$

The technology of Chirped Pulse Amplification $(\mathrm{CPA})^{26)}$ has made the ever shorter pulse and thus ever more compressed and thus higher intensity laser pulse. This progress has allowed for the LWFA method to become a widespread and common method that could overtake the conventional accelerator technology. Some of the results of LWFA indicate that the higher the intensity of the laser is, the 
shorter the response of the outcome (see Eq. [7]), which I coin as the Mourou Conjecture. ${ }^{56)}$ The LWFA method provides the path that makes it possible to realize the vision that the higher the power of the laser is, the higher the excitation of energy it can achieve and thus the pulse length that can encompasses is that much shorter. This vision is essentially the bedrock of what we discussed as the "relativistic engineering",22),23),57) and also what the attosecond science is finding now. ${ }^{58)}$ Our ability to control the coherent dynamics in relativistic regimes is so much enhanced by the advent of LWFA (see Fig. 9) and buffeted by the introduction of the CPA technique. These two methods can reinforce each other to reach ever shorter time scale and higher energy regimes in the future, rendering the Mourou conjecture a reality.

\section{Acknowledgments}

The author thanks a number of colleagues for collaborating with and advising us on the preparation of this work, who include: G. Mourou, F. Krausz, D. Habs, S. Karsch, L. Veisz, F. Gruener, M. Gross, P. Thirolf, F. Pfeiffer, R. Hörlein, K. Homma, H. Wu, E. Esarey, W. Leemans, N. Rostoker, F. Mako, H. Gies, T. Heinzl, R. Schützhold, M. Teshima, G. Dunne, A. Suzuki, F. Takasaki, K. Nakajima, A. Ogata, K. Yokoya, T. Tauchi, Y. Kato, T. Esirkepov, S. Bulanov, K. Kondo, M. Kando, M. Uesaka, L. M. Chen, Y. Fukuda, A. Pirozhkov, P. Bolton, A. Chao, P. Chen, T. Raubenheimer, C. Barty, C. Labaune, A. Giulietti, M. Downer, D. Jaroszynski, P. Shukla, R. Bingham, and F. Pegoraro. The author is very grateful for all these and others who have contributed to the development of laser acceleration in the past to realize the vision of collective acceleration and by that to create a new field of high field science. This paper is in part based on the author's Magistral Lecture at the ULIS (Ultraintense Laser Interaction Science Conference) 2009 at Frascati and in part on his Plenary Lecture of the Summer College of the Abdus Salam International Centre for Theoretical Physics, both on an occasion dedicated to commemorate the 30th anniversary of the publication of the laser acceleration paper in 1979.

\section{References}

1) Livingston, S.M. and Blewett, J.P. (1962) Accelerators. McGraw-Hill, NY.
2) Chao, A. and Tigner, M. (eds.) (1999) Handbook of Accelerator Physics and Engineering. World Scientific, Singapore.

3) Teng, L. (2001) Coherent principle of acceleration of charged particles. In Proc. Second Asian Part. Accel. Conf., Beijin, p. 926.

4) Tajima, T. (ed.) (1996) The Future of Accelerator Physics. AIP, NY.

5) Diamond, J. (2004) Collapse. Penguin, NY, 2004.

6) Veksler, V.I. (1956) CERN Symposium on High Energy Accelerators and Pion Physics. CERN, Geneva, p. 80.

7) Rostoker, N. and Reiser, M. (1978) Collective Methods of Acceleration. Harwood Academic, London.

8) Mako, F. and Tajima, T. (1984) Collective ion acceleration by a reflexing electron beam: model and scaling. Phys. Fluids 27, 1815-1820.

9) Tajima, T. and Mako, F. (1978) Self-consistent potential for a relativistic magnetized electron beam through a metallic boundary. Phys. Fluids 21, 1459-1460.

10) Tajima, T. and Dawson, J.M. (1979) Laser electron accelerator. Phys. Rev. Lett. 43, 267-270.

11) Tajima, T., Habs, D. and Yan, X.Q. (2009) Laser acceleration of ions for radiation therapy. Rev. Accel. Sci. Tech. 2, 201-228.

12) Tajima, T. (1985) High energy laser plasma accelerators, laser part. Beams 3, 351-413.

13) Ichimaru, S., Pines, D. and Rostoker, N. (1962) Observation of critical fluctuations associated with plasma-wave instabilities. Phys. Rev. Lett. 8, 231233.

14) Rostoker, N. and Rosenbluth, M.N. (1960) Test particles in a completely ionized plasma. Phys. Fluids 3, 1-14.

15) Ichimaru, S. (1973) Basic Principles of Plasma Physics. Benjamin, NY.

16) Kubo, R. (ed.) (1966) 1965 Tokyo Summer Lectures in Theoretical Physics. Benjamin, NY.

17) O'Neil, T. (1965) Collisionless damping of nonlinear plasma oscillations. Phys. Fluids 8, 2255-2262.

18) Dawson, J.M. (1959) Nonlinear electron oscillations in a cold plasma. Phys. Rev. 113, 383-387.

19) Bulanov, S.V. and Tajima, T. (2005) On the quasimonoenergetic electron beam generation in the laser wakefield acceleration. J. Part. Accel. Soc. Jpn. 2, 35-37.

20) Mourou, G.M., Tajima, T. and Bulanov, S. (2006) Optics in the relativistic regime. Rev. Mod. Phys. 78, 309-371.

21) Esarey, E. and Pilloff, M. (1995) Trapping and acceleration in nonlinear plasma waves. Phys. Plasmas 2, 1432-1436.

22) Bulanov, S., Esirkepov, T. and Tajima, T. (2003) Light intensification towards the Schwinger limit. Phys. Rev. Lett. 91, 085001.

23) Tajima, T. (2002) High field science and relativistic engineering. In Proc. Japan-Hungary Seminar (ed. Kanamori, J.). IIAS, Kyoto.

24) Esarey, E., Schroeder, S.B. and Leamans, W. (2009) Physics of laser-driven plasma-based electron accelerators. Rev. Mod. Phys. 81, 1229-1285. 
25) Chiu, C., Cheshkov, S. and Tajima, T. (2000) High energy laser-wakefield collider with synchronous acceleration. Phys. Rev. Special Topics-Accel. Beams 3, 101301.

26) Strickland, D. and Mourou, G. (1985) Compression of amplified chirped optical pulses. Opt. Comm. 56, 219-221.

27) Bulanov, S., Pegoraro, F., Pukhov, A. and Sakharov, A. (1997) Transverse-wake wave breaking. Phys. Rev. Lett. 78, 4205-4208.

28) Pukhov, A. and Meyer-ter-Vehn, J. (2002) Laser wakefield acceleration: the highly nonlinear brokenwave regime. Appl. Phys. B 74, 355-361.

29) Mangles, S.P.D., Murphy, C.D., Najmudin, Z., Thomas, A.G.R., Collier, J.L., Dangor, A. E. et al. (2004) Monoenergetic beams of relativistic electrons from intense laser-plasma interactions. Nature 431, 535-538.

30) Geddes, C.G.R., Toth, Cs., van Tilborg, J., Esarey, E., Schroeder, C.B., Bruhwiler, D. et al. (2004) High-quality electron beams from a laser wakefield accelerator using plasma-channel guiding. Nature 431, 538-541.

31) Faure, J., Glinec, Y., Pukhov, A., Kiselev, S., Gordienko, S., Lefebvre, E. et al. (2004) A laser-plasma accelerator producing monoenergetic electron beams. Nature 431, 541-544.

32) Leemans, W.P., Nagler, B., Gonsalves, A.J., Tóth, Cs., Nakamura, K., Geddes, C.G.R. et al. (2006) $\mathrm{GeV}$ electron beams from a centimetre-scale accelerator. Nature Phys. 2, 696-699.

33) Osterhoff, J., Popp, A., Major, Zs., Marx, B., Rowlands-Rees, T.P., Fuchs, M. et al. (2008) Generation of stable, low-divergence electron beams by laser-wakefield acceleration in a steady-state-flow gas cell. Phys. Rev. Lett. 101, 085002.

34) Schmid, K., Veisz, L., Tavella, F., Benavides, S., Tautz, R., Herrmann, D. et al. (2009) Few-cycle laser-driven electron acceleration. Phys. Rev. Lett. 102, 124801.

35) Hafz, N.A.M., Jeong, T.M., Choi, I.W., Lee, S.K., Pae, K.H., Kulagin, V.V. et al. (2008) Stable generation of GeV-class electron beams from selfguided laser-plasma channels. Nature Photon. 2, $571-577$.

36) Hosokai, T., Kinoshita, K., Ohkubo, T., Maekawa, A., Uesaka, M., Zhidkov, A. et al. (2006) Observation of strong correlation between quasimonoenergetic electron beam generation by laser wakefield and laser guiding inside a preplasma cavity. Phys. Rev. E 73, 036407.

37) Nakamura, K., Nagler, B., Tóth, Cs., Geddes, C.G.R., Schroeder, C.B., Esarey, E. et al. (2007) $\mathrm{GeV}$ electron beams from a centimeter-scale channel guided laser wakefield accelerator. Phys. Plasmas 14, 056708.

38) Brozek-Pluskab, B., Gliger, D., Halloua, A., Malka, V. and Gauduel, Y. (2005) Direct observation of elementary radical events: low- and high-energy radiation femtochemistry in solutions. Radiat. Phys. Chem. 72, 149-157.

39) Crowell, R., Gosztola, D., Shkrob, I., Oulianov, D.,
Jonah, C. and Rajh, T. (2004) Ultrafast processes in radiation chemistry. Radiat. Phys. Chem. 70, 501-509.

40) Fuchs, M., Weingartner, R., Popp, A., Major, Z., Becker, S., Osterhoff, J. et al. (2009) Laser-driven soft x-ray undulator source. Nature Phys. 5, 826-829.

41) Jaroszynski, D. and Rousse, A. (2009) Harnessing relativistic plasma waves as novel radiation sources from terahertz to X-rays and beyond (Proceedings Volume). Proc. SPIE 7359, pp. 1-308.

42) Pfeiffer, F., Weitkamp, T., Bunk, O. and David, C. (2006) Phase retrieval and differential phasecontrast imaging with low-brilliance X-ray sources. Nature Phys. 2, 258-261.

43) Yugami, N., Higashiguchi, T., Gao, H., Sakai, S., Takahashi, K., Ito, H. et al. (2002) Experimental observation of radiation from Cherenkov wakes in a magnetized plasma. Phys. Rev. Lett. 89, 065003.

44) Plateau, G.R., Matlis, N.H., Albert, O., Tóth, C., Geddes, C.G.R., Schroeder, C.B. et al. (2009) Optimization of $\mathrm{THz}$ radiation generation from a laser wakefield accelerator, AIP Conf. Proc. 1086, $707-712$.

45) Jackson, J.B., Mourou, M., Whitaker, J.F., Duling III, I.N., Williamson, S.L., Menu, M. and Mourou, G.A. (2008) Terahertz imaging for non-destructive evaluation of mural paintings, Opt. Comm. 281, 527-532.

46) Giulietti, A., Bourgeois, N., Ceccotti, T., Davoine, X., Dobosz, S., D'Oliveira, P. et al. (2008) Intense $\gamma$-ray source in the giant-dipole-resonance range driven by 10-TW laser pulses. Phys. Rev. Lett. 101, 105002.

47) Tajima, T. (1997) Prospect for compact medical laser accelerators. J. Jpn. Soc. Therap. Radiol. Oncol. 9 (Suppl. 2), 83-85.

48) Ogata, A., Kondoh, T., Norizawa, K., Yang, J. and Yoshida, Y. (2009) Collective energy loss of attosecond electron bunches. Jpn. J. Appl. Phys. 48, 056002 .

49) Tajima, T. and Chao, A. (2007) Patent applied, Beam Stopping and its Energy Recovery Using Plasma. Japan Patent 2007-314155 (filed December 5, 2007).

50) Raubenheimer, T. (2008) AAC presentation, Future R\&D for very high energy colliers. http:// aac08.1bl.gov/plenarytalks/PL02_Raubenheimer.pdf.

51) Mourou, G., Hulin, D. and Galvanauskas, A. (2006) The road to high peak power and high average power lasers: Coherent-Amplification-Network (CAN). AIP Conf. Proc. 827, 152-163.

52) HP: http://www.icuil.org/.

53) Xie, M., Tajima, T., Yokoya, K. and Chattopadyay, S. (1997) Studies of laser-driven $5 \mathrm{TeV} e^{+} e^{-}$colliders in strong quantum beamstrahlung regime. AIP Conf. Proc. 398, 233-242.

54) Suzuki, A. (2008) Outlook: Accelerator Science. ICFA, SLAC. http://www-conf.slac.stanford.edu/ icfa2008/Suzuki_103108.pdf.

55) Kawase, K., Kando, M., Hayakawa, T., Daito, I., Kondo, S., Homma, T. et al. (2008) Sub-MeV 
tunably polarized X-ray production with laser Thomson backscattering. Rev. Sci. Instr. 79, 053302 .

56) Mourou, G., AIP Proceedings LEI Conference (in press).

57) Pirozhkov, A.S., Ma, J., Kando, M., Esirkepov, T., Fukuda, Y., Chen, L.M. et al. (2007) Frequency multiplication of light back-reflected from a relativistic wake wave. Phys. Plasmas 14, 123106.

58) Krausz, F. and Ivanov, M. (2009) Attosecond physics. Rev. Mod. Phys. 81, 163-234.

(Received Dec. 15, 2009; accepted Feb. 8, 2010)

\section{Profile}

Professor Toshiki Tajima is considered as the founder of what is now called the emerging discipline of High Field Science and its leader in the world. He along with Dr. J. M. Dawson invented laser acceleration 30 years ago. This vision has been now experimentally verified and realized in many laboratories in the world. Applications of this ground breaking method include: ultrafast radiolysis, intraoperative radiation therapy, compact X-ray sources and free electron laser sources, bunch decelerators, and an alternative path toward the ultrahigh energy frontier. His work on laser acceleration and its demand on intense laser have prompted the spurt of High Field Science emergence and its impact on fundamental physics. He was the first to suggest the utility of laser driven electron and ion beams for the compact cancer therapy

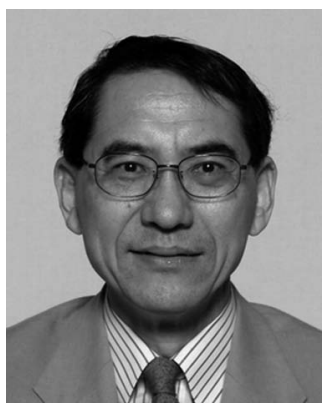
application and has heavily contributed to it. He serves as Chair of International Committee of Ultra Intense Lasers (ICUIL) under IUPAP and Chair of Scientific Advisory Committee of Extreme Light Infrastructure. He is the reciepeinet of the Blaise Pascal Chair. He was born on Jan. 18, 1948. He served, among others, as Jane and Roland Blumberg Professor of Physics at the University of Texas at Austin and Director General of Kansai Photon Science Institute, JAEA. 